\title{
Emotion Mimicry in Humanoid robots using Computational Theory of Perception
}

\author{
Mohsen Davoudi ${ }^{1}$, Mehdi Davoudi ${ }^{2}$ and Nima Seif Naraghi ${ }^{2}$ \\ 1. Politecnico di Milano, Milan, Italy \\ 2. Eastern Mediterranean University, Gazimagusa, Cyprus
}

\section{Introduction}

In robotics research one of the major issues is focused on the humanoids interaction modalities with humans. Humans have developed advanced skills in interpreting his emotions to the bodily expressions. If similar skills can be acquired by robots, it would allow them to generate behaviors that are familiar to us and thus increase their chances of being accepted as partners in our live.

It has been known that humans can express a lot of information and the emotional state of the performer using body movements. Human has some perception based behavior seen in his bodily expressions. Movements of hands, legs, head, etc show the inner emotion of the person in specific situations subconsciously. Reflecting the bounded ability of the human brain to resolve details of motions, perceptions are intrinsically imprecise.

The main goal of this Chapter is to introduce a novel approach for modeling an emotionbased motion generator for humanoids in order to present emotionally expressive styles of movements and emotion mimicry in different situations using Computational Theory of Perception (CTP).

Emotion mimicry is such an area, where expression of the proper emotion is based on a combination of various quantitative as well as qualitative measurements. In this perspective, a major shortcoming of existing approaches is that, as they are based on bivalent, they don't provide tools for dealing with perception-based information.

Humans have capability to perform a wide variety of physical motions without any measurement and computation. In performing such movements, humans employ perceptions of time, situation, possibility, inner emotions and other attributes of physical and mental objects. Motion characteristics in humans consist of measurement-based information and perception-based emotions. A big percent of human knowledge is perception-based.

The main goal of this Chapter is to introduce a novel approach for modeling an emotionbased motion generator for humanoids in order to present emotionally expressive styles of movements and emotion mimicry in different situations using CTP which contains a special capability to compute and reason with perception-based information.

A fuzzy logic-based analyzer that interprets the linguistic emotions that are common among people into what is called the Generalized Constraint Language (GCL) is introduced in this 
chapter. Fuzzy Logic-Based probability theory has the fundamental ability to operate on perception-based information, which bivalent logic-based probability theory does not posse. A particular GCL [1] is introduced for humanoid robots to categorize data needed for trajectory tracking for each joint of the robot which lead to emotion mimicry. An Artificial Neural Network generates velocity and acceleration for each joint of humanoid using GCL values as well. A concept that plays a key role in emotion mimicry with CTP is fuzzy logic based GCL.

An idea which underlies the approach described in this work is that an emotion may be viewed as a proposition that fuzzy analyzer approximates by means of the intensity and type of emotion. A proposition plays the role of a carrier of information. In our design fuzzy analyzer gets two types of linguistic proposition that are detected from a sentence:

1) The type of emotion such as happiness, anger, kindness, stress, surprise and sadness,

2) The intensity of emotion (very, more or less; extremely,...).

For example I say "move your hands very angry", two words are detected: angry and very. In the next step, an Artificial Neural Network (ANN) generates velocity and acceleration for each joint of humanoid using GCL variables. GCL is an interface between two portions of this analysis: a) emotion interpreter (the fuzzy analyzer), b) a data generator for physical motions of the humanoid joints. The overall structure of proposed robot motion system is shown in figure1.

The computational theory of perceptions enhances the ability of Artificial Intelligence (AI) to deal with real-world problems such as humanoid interactions in which decision relevant information is a mixture of measurements and perceptions. Motions in an intelligent humanoid are compromise between perceptions of emotions in common sense and measured data for trajectory tracking in humanoid joints.

In order to deal with perception-based analysis of the motion, an array of tools centering on Computing with Words and Perceptions (CWP), Computational Theory of Perceptions (CTP) and Precisiated Natural Language (PNL), are required[3].

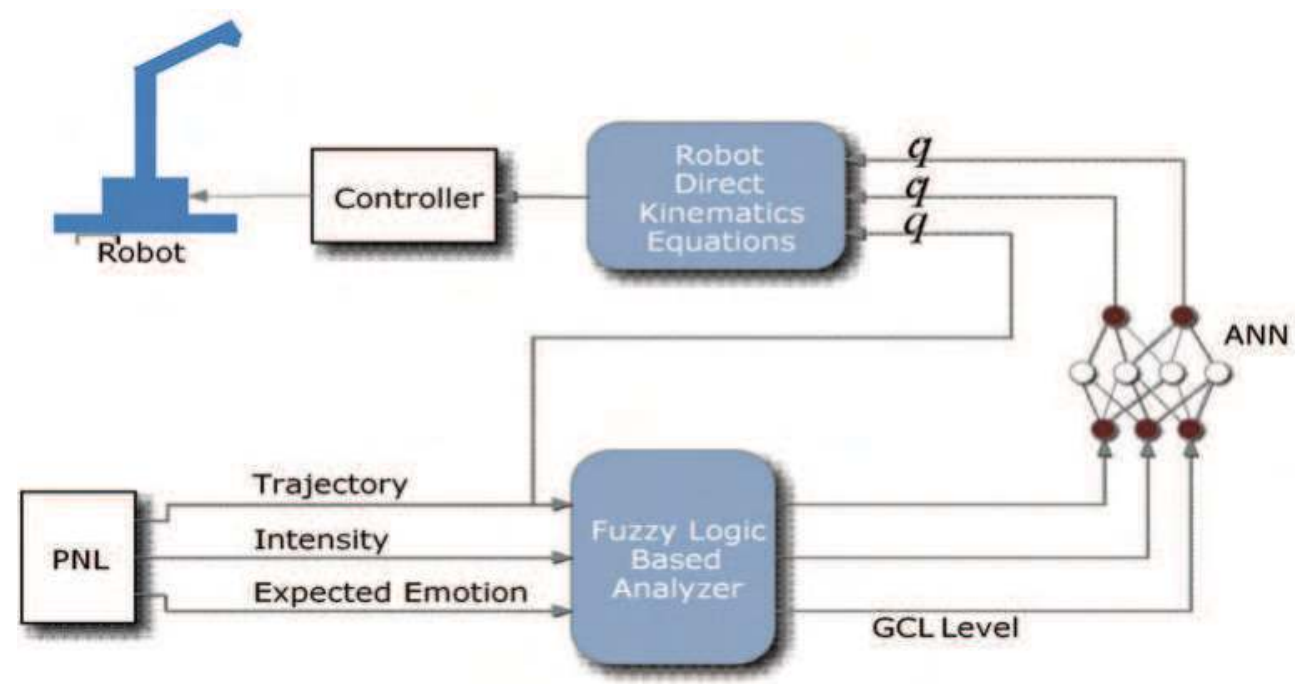

Fig. 1. The overall structure of intelligent robot motion system 


\section{Generalized Constrants for Intelligent Robot Motion}

CW (Computing with Words) serves two major purposes in intelligent robot motion generation: a) provides a machinery for dealing with emotions in which precise information is not available, b) provides a machinery for dealing with emotions in which precise information is available, but there is a tolerance for imprecision which can be exploited to achieve tractability, robustness, simplicity and low solution cost.

Precisiated Natural Language (PNL) is a sublanguage of precisiable propositions in Natural Language (NL) which is equipped with two dictionaries: 1) NL to GCL, 2) GCL to PFL (Protoform Language) and a set of rules of deduction (rules of generalized constrained propagation) expressed in PFL.

Perceptions in Emotion mimicry are f-granular in the sense that a) the boundaries of perceived classes are fuzzy and b) the values of perceived attributes are granular, with a granule being a clump of values drawn together by indistinguishability, similarity, proximity or functionality of emotions.

$$
\text { p=proposition in a natural language }
$$

A proposition is a perception if it contains a) fuzzy quantifiers such as many, most, few, etc., b) fuzzy qualifiers such as usually, probably, possibly, typically, generally,... c) fuzzy modifiers such as very, more or less, extremely,... and d) fuzzy nouns, adjective or adverbs such as emotion nouns that is prevalent among people in a culture. We considered a number of inner emotions in this paper: Angriness, kindness, stress, happiness.

Generally, the mathematics of perception-based information has a higher level of generality than the mathematics of measurement-based information. The constrained variable, $X$, may assume a variety of forms. In particular,

$X$ is an n-array variable, $X=\left(X_{1}, \ldots, X_{n}\right)$

$X$ is a proposition, e.g., $X=$ very angry

$X$ is a function

$X$ is a function of another variable, $X=f(Y)$

$X$ is conditioned on another variable, $X / Y$

$X$ has a structure, e.g., $X=$ Emotion(Stress)

$X$ is a group variable. In this case, there is a group, G[A]; with each member of the group, Name $_{i}, i=1, \ldots, n$, associated with an attribute-value, $A_{i} . A_{i}$ may be vector-valued. Symbolically

$$
\text { G[A]: Name } \mathrm{N}_{1} / \mathrm{A}_{1}+\ldots+\mathrm{Name}_{n} / A_{n}
$$

$G[A]$ is a relation $(r)$ and $\mathrm{X}$ is a generalized constraint [4].

Possibilistic of emotion mimicry ( $r=$ blank) is written such as equation 3.

$$
X \text { is } R
$$

which $R$ playing the role of the possibility distribution of $X$. 
For example:

$$
X \text { is }[a, b]
$$

means that $[a, b]$ is the set of possible values of $X$. Another example:

$$
X \text { is "happy" }
$$

In this case, the fuzzy set labeled happy is the possibility distribution of $X$. If $\mu_{\text {small }}$ is the membership function of happy, then the semantics of $X$ is "happy" is defined by

$$
\operatorname{Poss}\{X=h a p p y\}=\mu_{\text {small }}(\text { happy })
$$

where $u$ is a generic value of $X[6]$. Probabilistic in emotion mimicry $(r=p)$

$$
X \text { isp } R
$$

which $R$ playing the role of the probability distribution of $X$. For example:

$$
X i s p N\left(m, \sigma^{2}\right)
$$

Means that $X$ is a normally distributed random variable with mean $\mathrm{m}$ and variance $\sigma^{2}$. For example, if $X$ is a random variable which takes values in a finite set of emotions $\left\{e_{1}, \ldots\right.$, $\left.e_{n}\right\}$ with respective probabilities $p_{1}, \ldots, p_{n}$, then $X$ may be expressed symbolically as equation 9:

$$
X \text { isp }\left(p_{1} \backslash e_{1}+\ldots+p_{n} \backslash e_{n}\right)
$$

with the semantics

$$
\operatorname{prob}\left(X=e_{i}\right)=p_{i}, \quad i=1, \ldots, n
$$

For $e_{i}=$ happy probability of $X$ is given in equation 11 :

$$
\operatorname{prob}(X \text { is happy })=\int \mu_{\text {small }}(u) f(u) d u
$$

Hence, test-score of this constraint on $f$ is given by:

$$
t s(f)=\mu_{\text {likely }}\left(\int f(u) \mu_{\text {happy }}(u)\right)
$$


As other consideration, Bimodal $(r=b m)$ is the bimodal constraint is symboled in equation 13 ,

$$
X \text { isbm R, }
$$

$R$ is a bimodal distribution of the form:

$$
R: \quad \Sigma_{i} k_{i} \backslash A_{i} \quad, i=1, \ldots, n
$$

this means that

$$
\operatorname{Prob}\left(\mathrm{X} \text { is } A_{i}\right) \text { is } k_{i}
$$

\section{Example:}

$$
\operatorname{prob}(\text { Emotion(happy) is High) }
$$

is likely

$$
\int g\left(e_{4}\right) \mu_{h i g h}\left(e_{4}\right) d e_{4}
$$

or, in annotated form,

$$
G C(g)=X / \int g(e) \mu_{\text {high }}(e) d e \text { is R/likely }
$$

the test-score of this constraint on $f$ and $g$ is given by

$$
\begin{aligned}
& t s(f)=\mu_{\text {likely }}\left(\int f(u) \mu_{\text {high }}(u) d u\right) \\
& t s(g)=\mu_{\text {likely }}\left(\int g(u) \mu_{\text {high }}(u) d u\right)
\end{aligned}
$$

Basically, we use two forms of $X$ :

1) proposition form expressed as $p$,

2) function form (measure-based information) expressed as $f$. 


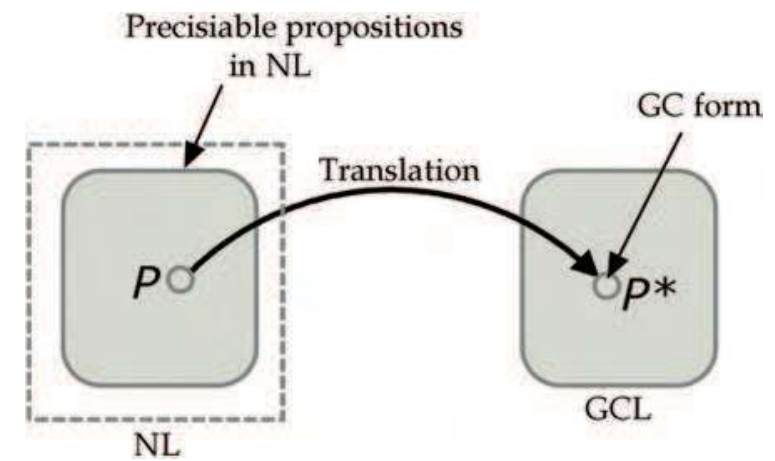

Fig. 2. GCL form of proposition expressed as $p$

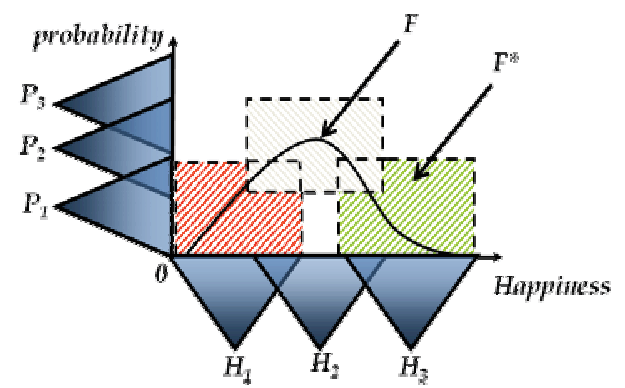

Fig. 3. Fuzzy-graph constraint. $f^{*}$ is a fuzzy graph which is an approximate representation of function form (measure-based information) expressed as $f$

Considering figure3 we can express the probability of happiness as given in equation 16:

$$
P(\text { happiness })=P 1 \backslash H 1+P 2 \backslash H 2+P 3 \backslash H 3
$$

\section{Fuzzy Analyzer}

We supposed four emotions that are common among people:

$$
U=\left\{e_{1}, e_{2}, e_{3}, e_{4}\right\}
$$

$e_{1}:$ Kindness $(k)$

$e_{2}$ : Stress (s)

$e_{3}$ : Angriness (a)

$e_{4}$ : Happiness $(h)$

$$
\text { Decision }=\left\{\begin{array}{l}
\text { Offensiveness }=f\left(e_{i}\right), \quad i=1 \ldots 4 \\
\text { Intensity }=g\left(e_{i}\right), \quad i=1 \ldots 4
\end{array}\right.
$$


Membership functions of offensiveness:

$$
\mu_{o}(i)=\sup _{e}\left(\mu_{o}\left(\int_{U} \mu_{e i}(u) f(u) d u\right)\right)
$$

Membership functions of intensity:

$$
\mu_{i}(i)=\sup _{e}\left(\mu_{o}\left(\int_{U} \mu_{e i}(u) g(u) d u\right)\right)
$$

An Example: John is so much happy today. He wants to play guitar.

Combined propositions are: (happy + act of playing guitar). Deduction with perceptions involves the use of protoformal rules of generalized constraint propagation. Playing guitar is an action that is done using organs specially hands. Therefore considering his inner emotion, his movement is so quick and we can guess his inner emotion (happiness) from the motions of his hands. As shown in table 1 and table 2, a phrase is converted to prototype form.

\begin{tabular}{|c|c|}
\hline \hline proposition in NL & precisiation \\
\hline$p$ & $p^{*}$ (GC-form) \\
\hline John is very happy & $\begin{array}{c}\text { ECount (intensity. happiness } \\
\text { /happiness) is much }\end{array}$ \\
\hline
\end{tabular}

Table 1. Proposition interpretation

\begin{tabular}{|c|c|}
\hline precisiation & protoform \\
\hline \hline$p^{*}$ (GC-form) & $P F\left(p^{*}\right)$ \\
\hline \hline $\begin{array}{c}\Sigma \text { Count (intensity. happiness } \\
\text { happiness) is much }\end{array}$ & $\mathrm{Q} A^{\prime}$ s are $B^{\prime} \mathrm{s}$ \\
\hline \hline
\end{tabular}

Table 2. Precisiation interpretation

After parsing $p$ is converted to semantic parse that may be one of these forms:

1) Logical form

2) Semantic network

3) Conceptual graph

4) Canonical form

Semantic parse can be abstracted to more than one protoforms. In order to computation of GCL form for emotion sets we use fuzzy analyzer as inference motor. 


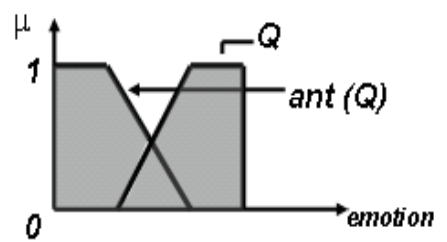

(a)

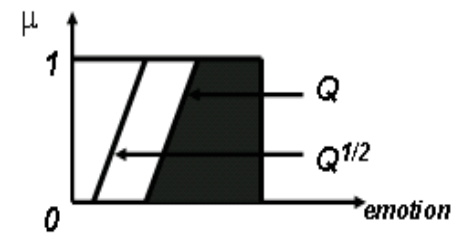

(b)

Fig. 4. Emotion definition, A simple fuzzification for happiness (a), culture variation is modeled as shift in the membership function (b).

A simple fuzzification for happiness is shown in figure 4 (a). $\mu$ is the membership function of the intensity. Naturally, $Q$ is happiness and ant $(Q)$ is sadness. The definition of happiness may be changed by cultures which is modeled as a shift in the membership functions (see figure4 (b)). The overall scheme of the fuzzy analyzer is demonstrated in figure 5.

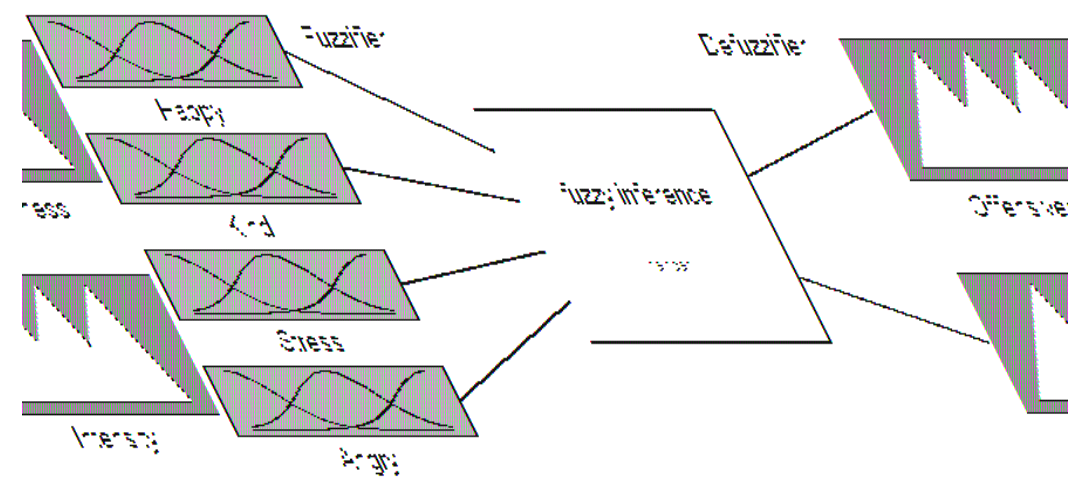

Fig. 5. Fuzzy analyzer

A standard GCL for humanoid robots is defined to categorize data needed for trajectory tracking for each joint which leads to emotion mimicry and intelligent robot motion. As discussed in section2, motion characteristics in humans consist of measurement-based information and perception-based emotions and a big percent of human knowledge is perception-based. Expected mimicry or right motion expression in each situation is the goal that we consider to design humanoid robots. So defining some emotion based rules for fuzzy analysis and creation of deduction database for emotion definition is a base for designing. (see figure 6) 


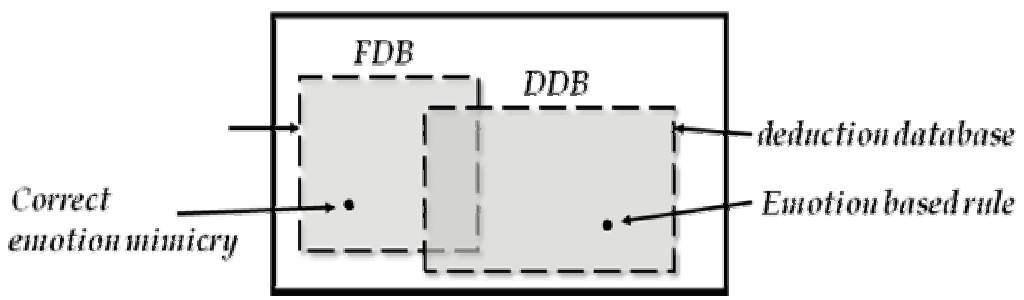

Fig. 6. Database interaction

Information about trajectory of each joints in a humanoid, the time that motions accrue and the supposed age for the humanoid are measurement-based information that can be retrieved from sensors and the motion generator algorithm in a humanoid. They are going to be given to an artificial neural networks (ANN) for motion generation.

The offensiveness and intensity variables which are GCL form of perception-based information are the other inputs of ANN as well.

The last step for mimicry is the generation of the movements from the GCL variables. Using an ANN with less than 30 neurons GCL form is translated to $\dot{q}, \ddot{q}$ and delay time value for each joint of humanoid. Figure 7 shows the structure of artificial neural networks designed for motion generation.

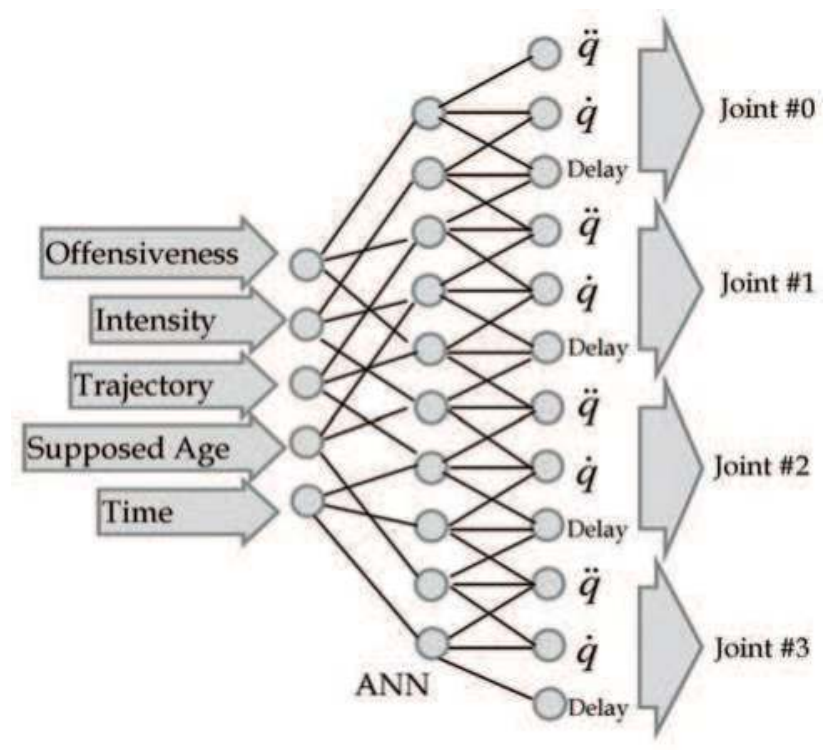

Fig. 7. Artificial Neural Networks

Multi Layer Perceptron (MLP), RBF and Recurrent Neural Networks are candidate networks for this purpose. For simplicity, a MLP is used as the core of ANN. (See figure 8) 


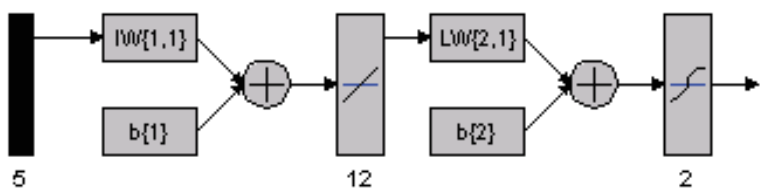

Fig. 8. Multi Layer Perceptron (MLP) as the core of ANN

\section{Simulation Results}

In this section a computer based simulation for control of a joint in a humanoid robot is demonstrated. Figure 9 shows the state in which the robot asked to move his arm kindly. Three curves with different amplitude and phase shows the intensity of the kindness.

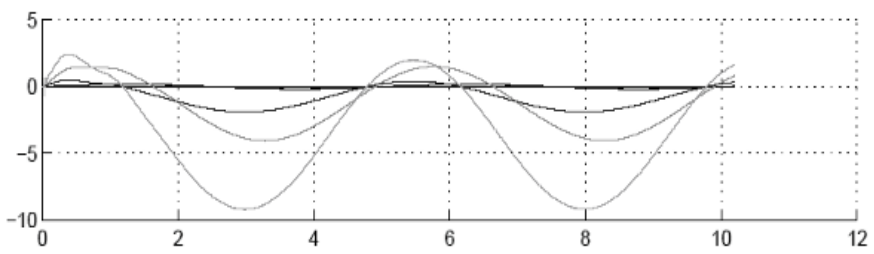

Fig. 9. Joint position control in kindness state

Another emotion that has been modeled in the simulation is angriness which is shown in figure10. In this figure the generated motions of an angry person is presented as sharp curves in control signal. Roughly, the intensity parameter effects on the control signal as increment of amplitude of control signal (speed of the motion).

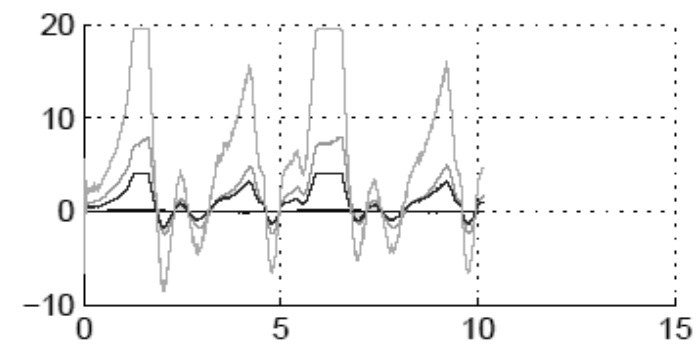

Fig. 10. Joint position control in angriness state

Since the mimicry of kindness is logically smoother than mimicry of angriness in the joint motions, comparing curves of figure 9 and figure 10 visually we can infer that the joint motions in kind state are more smoother.

figure 11 shows the practical result of the joint response to the position control signal tracking the desired position curve. The physical delay of trajectory tracking in humanoid robot's joints may cause some problems in emotion mimicry which can be modeled in the 
emotion mimicry system as well. Neglecting the differences in desired value and joint response in the joints and delays, the whole concept of the emotion mimicry is protected.

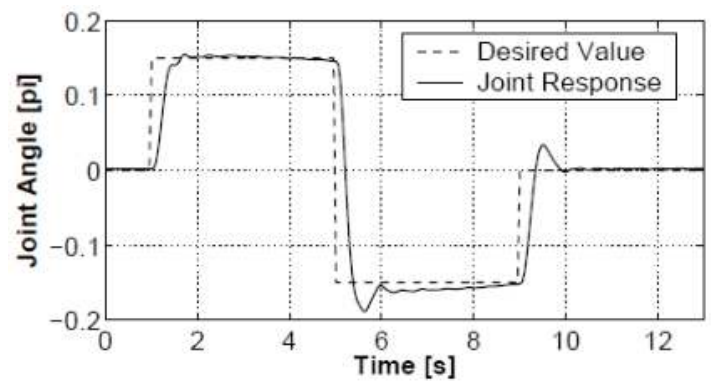

Fig. 11. Joint response to the position control signal

\section{Conclusion}

Emotion mimicry is such an area, where presentation of the proper emotion is based on a combination of various quantitative as well as qualitative measurements. In this perspective, a major shortcoming of existing approaches is that, based as they are on bivalent, they don't provide tools for dealing with perception-based information. It is not possible to construct a computational theory of perceptions within the conceptual structure of bivalent logic and probability theory in this area. With CTP and GCL using fuzzy logic we solved problems of existing approaches which neural networks and/or fuzzy logic can't solve lonely.

\section{References}

A. Bardossy, L. Duckstein(1995), Fuzzy Rule-based Modelling with Application to Geophysical, Biological and Engineering Systems, CRC Press

D. Dubois, H. Prade(1992), Gradual inference rules in approximate reasoning, Information Sciences: An International Journal 61, 103-122

D. Dubois, H. Fargier, H. Prade(1993), The calculus of fuzzy restrictions as a basis for flexible constraint satisfaction, in: Proc. 2nd IEEE Int. Conf. on Fuzzy Systems, San Francisco, CA, pp. 1131-1136

D. Dubois, H. Prade(1996), Fuzzy Information Engineering: a Guided Tour of Applications, John Wiley and Sons

D. Filev, R.R. Yager(1994), Essentials of Fuzzy Modeling and Control, Wiley-Interscience

M. Higashi, G.J. Klir(1993), Measures of Uncertainty and Information Based on Possibility Distributions, Fuzzy Sets for Intelligent Systems, Morgan Kaufmann Publishers, San Mateo, CA, pp. 217-232

M. Jamshidi, A. Titli, L.A. Zadeh, S. Boverie, (1997), Applications of Fuzzy Logic towards High Machine Intelligence Quotient Systems, Environmental and Intelligent Manufacturing Systems Series, vol. 9, Prentice Hall, Upper Saddle River, NJ

A. Kaufmann, M.M. Gupta(1985), Introduction to Fuzzy Arithmetic: Theory and Applications, Von Nostrand, New York

E.H. Mamdani, S. Assilian(1975), An experiment in linguistic synthesis with a fuzzy logic controller, International Journal of Man-Machine Studies,1-13. 
V. Novak (1999), I. Perfilieva, J. Mockor, Mathematical Principles of Fuzzy Logic, Kluwer, Boston/ Dordrecht

H.T. Nguyen(1993), On Modeling of Linguistic Information Using Random Sets, Fuzzy Sets for Intelligent Systems, Morgan Kaufmann Publishers, San Mateo, CA, pp. 242-246

T.J. Ross(2004), Fuzzy Logic with Engineering Applications, second ed., Wiley

J. Yen, R. Langari(1998), Fuzzy Logic: Intelligence, Control and Information, first ed., Prentice Hall, Berlin

L.A. Zadeh(1975), The concept of a linguistic variable and its application to approximate reasoning, Part I: Information Sciences, 199-249

L.A. Zadeh (1983), A computational approach to fuzzy quantifiers in natural languages, Computers and Mathematics,149-184

L.A. Zadeh(2002), toward a perception-based theory of probabilistic reasoning with imprecise probabilities, Journal of Statistical Planning and Inference 105 


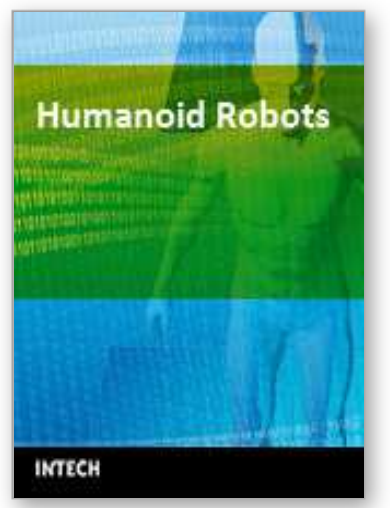

\author{
Humanoid Robots \\ Edited by Ben Choi
}

ISBN 978-953-7619-44-2

Hard cover, 388 pages

Publisher InTech

Published online 01, January, 2009

Published in print edition January, 2009

Humanoid robots are developed to use the infrastructures designed for humans, to ease the interactions with humans, and to help the integrations into human societies. The developments of humanoid robots proceed from building individual robots to establishing societies of robots working alongside with humans. This book addresses the problems of constructing a humanoid body and mind from generating walk patterns and balance maintenance to encoding and specifying humanoid motions and the control of eye and head movements for focusing attention on moving objects. It provides methods for learning motor skills and for language acquisition and describes how to generate facial movements for expressing various emotions and provides methods for decision making and planning. This book discusses the leading researches and challenges in building humanoid robots in order to prepare for the near future when human societies will be advanced by using humanoid robots.

\title{
How to reference
}

In order to correctly reference this scholarly work, feel free to copy and paste the following:

Mohsen Davoudi, Mehdi Davoudi and Nima Seif Naraghi (2009). Emotion Mimicry in Humanoid Robots Using Computational Theory of Perception, Humanoid Robots, Ben Choi (Ed.), ISBN: 978-953-7619-44-2, InTech, Available from:

http://www.intechopen.com/books/humanoid_robots/emotion_mimicry_in_humanoid_robots_using_computatio nal_theory_of_perception

\section{INTECH}

open science | open minds

\section{InTech Europe}

University Campus STeP Ri

Slavka Krautzeka 83/A

51000 Rijeka, Croatia

Phone: +385 (51) 770447

Fax: +385 (51) 686166

www.intechopen.com

\section{InTech China}

Unit 405, Office Block, Hotel Equatorial Shanghai

No.65, Yan An Road (West), Shanghai, 200040, China

中国上海市延安西路 65 号上海国际贵都大饭店办公楼 405 单元

Phone: +86-21-62489820

Fax: $+86-21-62489821$ 
(C) 2009 The Author(s). Licensee IntechOpen. This chapter is distributed under the terms of the Creative Commons Attribution-NonCommercialShareAlike-3.0 License, which permits use, distribution and reproduction for non-commercial purposes, provided the original is properly cited and derivative works building on this content are distributed under the same license. 